\title{
La incidencia de las pausas activas en la callidad de vida laboral de la empresa Maxi-Distribuciones
}

\section{The impact of the active brealks in the quallity of working life now Maxi-Distribuciones}

GARDENIA VANESA GONZÁLEZ FRANCO Consultora independiente

Correo electrónico: gardegonzalez@yahoo.es

LUIS ANIIBAL YEROVII VILILALBA Universidad Tecnológica Equinoccial. Quito Correo electrónico: luis.yerovi@ute.edu.ec

LUIS ENRIQUE BARRENO BENAVIDES

Universidad Tecnológica Equinoccial. Quito Correo electrónico: 1barreno@ute.edu.ec

RECIBIIDO: 27 marzo 2015 / APROBADO: 29 mayo 2015

\section{Resumen}

El artículo analiza la incidencia de las pausas activas en los trabajadores de la empresa Maxi- Distribuciones operadora del servicio de radiocomunicación, el artículo parte con la descripción teórica de las pausas activas, así como de los elementos que la integran como son: salud laboral, salud en el trabajo y calidad de vida laboral, desde las perspectivas de diferentes autores; igualmente experiencias en la aplicación de las pausas activas en nuestro país; se analiza además, la situación actual de las mismas en la empresa sujeto de investigación, a partir de ello se propone un programa de pausas activas, basado en una rutina de ejercicios, tendientes a eliminar y disminuir los problemas causados por la rutina en el trabajo sin ninguna pausa o terapia; finalmente, se realiza una evaluación de los resultados obtenidos luego de la aplicación del programa señalado a los trabajadores de la empresa 
PALABRAS CLAVE: pausas activas, salud laboral, calidad de vida laboral, rutina de ejercicios.

\begin{abstract}
Albstract:
The article analyzes the impact of active breaks in company workers Maxi operator Distributions radio communication service, the article starts with the theoretical description of the active breaks, as well as the elements that integrates as: occupational health, occupational health and quality of working life, from the perspectives of different authors; also experiences in implementing active breaks in our country; it also analyzes the current situation of the same in the enterprise research subject, on this basis a program of active breaks, based on an exercise routine, aimed at eliminating and reducing problems caused by routine work proposed without pause or therapy; Finally, an evaluation of the results obtained after implementing the program pointed to company employees is done.
\end{abstract}

KEYWORDS: active breaks, occupational health, quality of working life

CLASIIFICACIÓN JEL: I31-L23-M54

\section{Introducción}

La base para mejorar y mantener la productividad de los bienes y servicios en las empresas y organismos, en general, es el mantenimiento de la fuerza laboral saludable, lo que se logra mediante la realización de las pausas activas dentro de la jornada laboral de los trabajadores. La implementación de una rutina de ejercicios para cada grupo de trabajadores, permite mantenerlos activos y saludables, lo que facilita mejorar sustancialmente su concentración y rendimiento. De allí la importancia de realizar una investigación en el campo práctico de una empresa ecuatoriana como es el caso de Maxi- Distribuciones, operadora del servicio de radiocomunicación.

Dentro de los procesos básicos de la administración y gestión de talento humano, varios especialistas coinciden con el proceso de mantenimiento y retención del personal, obviamente, cada uno tiene su propio estilo para denominarlo; lo importante, es la finalidad que tiene este proceso, que es la satisfacción del personal con su trabajo y con la organización a la que pertenece, y que promueva su permanencia en el mismo (Delgado J, 2014).

Con este antecedente, la base teórica de este estudio, se fundamentó en las principales vertientes del pensamiento epistemológico, que han venido aportado diversos autores sobre el tema propuesto, para lo cual, se analizó en primera instancia la salud laboral, pasando a identificar algunos problemas de salud en el trabajo, programas de promoción y prevención de salud laboral, definición de la técnica de las pausas activas y también, la incidencia de esta en la calidad de vida laboral, finalizando con la empresa Maxi-Distribuciones. 


\section{Salud laboral}

En lo que compete a trabajo y salud laboral existen múltiples aportaciones, tanto a su concepto, como al criterio que abarca este tema; por lo tanto se elaborará un resumen de las distintas respuestas y acotaciones de los autores.

Realizando una retrospectiva, se tiene que, en 1970 se perseguía el cambio en el comportamiento del modo de vida del individuo, en 1980, el centro de atención era el individuo y los programas de salud y, a partir de 1990 impera la necesidad de combinar la salud del trabajador, con un lugar de trabajo que promueva salud (Salud O. M., 2003).

Parra M, (2003) señala que, un país mejora el nivel de salud de su población, cuando asegura que todas las personas en condiciones de trabajar puedan acceder a un empleo que satisfaga, no sólo sus necesidades económicas básicas, sino que llene otros aspectos positivos del trabajo.

Acertadamente, este autor intuye que un país saludable, es el que asegura el pleno empleo a todas aquellas personas que estén en condiciones de trabajar; también, menciona que un empleo además, de satisfacer necesidades económicas "básicas, deberá, satisfacer otros aspectos, como son: salario, actividad física y mental; contacto social, desarrollo de una actividad con sentido y producción de bienes y servicios necesarios para el bienestar de otros individuos y grupos (Parra M, 2003).

Según la Organización Mundial de la Salud (OMS, 2014) la salud laboral, se preocupa de la búsqueda del máximo bienestar posible en el trabajo, tanto en la realización del trabajo como de las consecuencias de este, en todos los planos, físico, mental y social.

Esta definición sintetiza, el buscar las maneras posibles para conseguir el máximo bienestar en el trabajo, para lo cual, se deberá tomar medidas que promuevan la salud y prevengan daños al efectuar el trabajo.

Según Mazzafero E. (1999) una fuerza de trabajo saludable no solo contribuye a la productividad y riqueza del país, sino a la motivación, satisfacción y calidad de vida de la población. Además Salud O. M., (1995) señala que el trabajo puede tener efectos positivos y adversos para la salud del trabajador, aproximadamente entre un 30 y $50 \%$ de los trabajadores reportan estar expuestos a altos riesgos físicos, químicos, biológicos o inexplicables sobrecargas de trabajo pesado, factores ergonométricos que son una amenaza para su salud y su capacidad de trabajo; otro tanto, presenta altos síntomas de estrés producidos por sobrecarga psicológica y laboral.

Según estos autores, son cuantiosos los efectos que se producen en la salud de los trabajadores; positivos como es potenciar la satisfacción, motivación, bienestar y estilos saludables dentro del trabajo; o negativos que disminuyan la productividad y refuercen la indignación y perdida de valiosos talentos; está en las manos de los trabajadores, unidades de talento humano y directivos tomar cartas en el asunto.

\section{Problemas de sallud en ell trabajo}

Hoy por hoy, el ser productivos y competitivos entre las organizaciones es puntal de modernización y vanguardia. Los trabajadores para ir de la mano con la organización han tenido que acoplarse al ritmo del trabajo, a las condiciones del lugar y al ambiente laboral. Pero estos ajetreos intensifican los problemas de salud en el trabajador llevándolo a extremos, como es el sedentarismo y el exceso de carga laboral física y mental, rompiéndose la armonía del cuerpo, mente y de reacción con lo que le rodea. 
Parra (2003) define a la carga de trabajo como el conjunto de requerimientos físicos y mentales al que se ve sometido el trabajador para realizar una tarea, considerando este factor de alto riesgo laboral, ya que contempla dos aspectos: la demanda de esfuerzo físico y la demanda de esfuerzo mental o psicológico del trabajo.

Es inevitable que este aspecto laboral puede causar daño en la salud del trabajador, el exceso de esfuerzo físico y/o mental, denotará: fatiga, cansancio, malas posturas, posturas estáticas, fuerzas indebidas etc.; acelerando, la aparición de problemas musculo esqueléticos (físico) y trastornos mentales como: depresión, estrés, agotamiento y otros malestares.

La Organización Internacional del Trabajo (OIT, 2010) define a las enfermedades profesionales como aquellas contraídas por la exposición a factores de riesgo, resultado de la actividad laboral. Las clasifica de acuerdo a la causa que, puede ser por agentes químicos y físicos, o que afecten al sistema óseo muscular, respiratorio, etc. Para Guerrero (2004) entre las enfermedades más comunes que atentan a la salud y bienestar del trabajador están: fatiga visual, estrés, síndrome del túnel carpiano y trastornos de trauma acumulativo. Está en las unidades de talento humano y en los altos mandos prevenir y promocionar alternativas que reduzcan los riesgos que las provocan, y a los que están expuestos los trabajadores en el lugar de trabajo.

Adicionalmente Mossink (2004) manifiesta que los trastornos músculo esqueléticos son afecciones al aparato locomotor, es decir, músculos, tendones, esqueleto óseo, cartílagos, ligamentos y nervios; estos pueden ser irrelevantes en sus inicios pero permanentes e irreversibles en el tiempo, provocando incapacidad en el individuo.

Estos problemas de salud en el trabajo acarrean altos índices de absentismo laboral, desmotivación, insatisfacción y elevados costos de seguro por tratamientos; lo cual se podría reducir si se promovieran hábitos saludables dentro de la jornada laboral.

En Ecuador, no son desconocidas estas definiciones en lo concerniente a problemas laborales, Herrera (E1 Comercio,2015) informa que enfermedades como lumbalgia crónica (dolor en la espalda baja), hernia discal (dolencias en la columna vertebral), síndrome del túnel carpiano (presión sobre los nervios que trasmiten a la muñeca), lumbalgia y hombro doloroso que es uno de los casos de tendinitis suman el $69 \%$ de las enfermedades laborales reportadas en el 2012, se han prolongado en el personal, que utiliza constantemente el computador, sea este administrativo u operativo.

\section{Programas de promoción y prevención de sallud laborall}

La descompensación de la salud, no solo afecta al individuo sino, al grupo de trabajo y por ende a la organización, según Logroño (2011) el promover el trabajo sano y seguro, logra habilitar a los trabajadores para que lleven vidas social y económicamente productivas, contribuyendo al desarrollo, permitiendo el enriquecimiento humano y profesional en el trabajo.

Si se desea una organización sana, "más vale prevenir que lamentar" y es obligación del área de talento humano, establecer programas que permitan retener al personal; entre estos están los relacionados con la salud, y si lo vemos desde este punto de vista organizacional, los hábitos saludables generarán, bienestar a todos los que integran la organización.

Francesca Trejo, responsable del servicio de prevención del grupo NOVARTIS en España, opina que la promoción de la salud en la organización es importante, porque mejora la integración, motivación y bienestar del trabajador, además 
reduce el absentismo, impulsa la competitividad y genera un buen ambiente global en las empresas, lo puede reducir hasta en un $40 \%$ la incidencia de las enfermedades no transmisibles asociadas a hábitos no saludables (Salud, 2013).

\section{Pausas activas}

Según Castro (2011) la gimnasia laboral o pausas activas es una técnica que mediante ejercicios preparatorios y compensatorios, permiten al individuo liberar tensión de carácter fisiológico y psicológico generado por el trabajo en rutinas de recuperación de 15 minutos diarios. Para Castro, realizar actividad física en un tiempo determinado, durante el trabajo, mantendrá al cuerpo y la mente libre de tensiones, distrayéndolo y rompiendo la rutina laboral.

La gimnasia laboral incluye ejercicios específicos de estiramiento, fortalecimiento muscular, relajación y coordinación motora, celebrada en diferentes sectores o departamentos de la empresa; su principal objetivo es prevenir y reducir los casos de lesiones por esfuerzos repetitivos o disturbios óseo musculares relacionados con el trabajo, también enfatiza que la gimnasia laboral puede ser compensatoria, preparatoria y de relajamiento (Oliveira J. , 2006).

Además Castro (2011) agrega que, dentro de estos periodos de descanso, las personas realizan una serie de actividades y acciones que les permite a diferentes partes del cuerpo, un cambio en su rutina habitual, con el fin de prevenir la aparición de problemas o desórdenes en diferentes grupos musculares y articulares, además, de reactivar o mejorar la atención y la producción en la realización de las tareas.

Es interesante que una organización cuente con esta técnica para afianzar y reforzar los estilos de vida saludable, inyectando una alternativa que rompa la monotonía, inculcando la sensibilización de tener cuerpo y mente sanas; previniendo posibles dolencias al sistema locomotor, bajas de autoestima por sobrepeso y una inadecuada alimentación por falta de un balance nutritivo, o por comer poco o nada, y fuera de las horas establecidas para esta actividad.

La técnica de las pausas activas ha sido desarrollada en empresas tanto del sector público como del privado. En el Ecuador, estas iniciativas están dando sus primeros pasos con el programa "Agita tu mundo" que se lo lleva a cabo en las zonales y distritos del Ministerio de Salud Pública desde el año 2013. Este programa, contiene prácticas saludables como: pausas activas, bici saludable y fructívoros; por el momento no existen resultados oficiales de estas prácticas, sin embargo, según información proporcionada por la Ing. Margarita Cañar (Ministerio de Salud) y encargada de llevar a cabo este programa, ha manifestado que gracias a este, el rendimiento de cada uno de los trabajadores en su puesto de trabajo, se ha incrementado, así como su actitud para con el desempeño del mismo (Ministerio de Salud, 2013).

En la misma línea de acción de acuerdo a la página web del Ministerio del Deporte, en el link de noticias año 2012-Esmeraldas, inició el proyecto denominado "Pausa Activa Laboral", que consiste en una rutina de estiramientos y ejercicios realizados por funcionarios de distintas instituciones públicas del sector durante su jornada laboral por 15 minutos diarios (Ministerio del Deporte, 2012); desde luego, estas experiencias son puntuales y aún no se han hecho extensivas a todo el sector público, se desconoce casos documentados en las empresas privadas ecuatorianas.

Situación actual de las pausas activas en la empresa Maxi-Distribuciones

Para recolectar información y datos se aplicó la observación y entrevistas que, son técnicas de investigación de campo. La empresa MaxiDistribuciones al contar con un total de 31 trabajadores, el criterio a desarrollar fue el censo, considerando a todo el personal como universo. 
Como instrumento de identificación y evaluación de los factores de riesgo, se utilizó un diagrama de decisión, ficha de datos generales y lista de chequeo propuesto por Cerda (2012) que tiene como objetivos:

- Identificar los factores de riesgo relacionados con movimientos repetitivos, postura, movimiento, solo fuerza y tiempos de recuperación o descanso.

- Evaluar preliminarmente el nivel de riesgo en los puestos de trabajo,

- Obtener información que sirva para la corrección de los factores de riesgo identificados.

- Obtener información para elaboración de programas de promoción y prevención en salud laboral.

Con la observación directa de la tarea realizada en el momento de la evaluación y entrevista, con preguntas al trabajador, se obtuvo información en tiempo real y en las condiciones habituales de trabajo a través de la lista de chequeo para identificar los siguientes factores de riesgo.
Los pasos a observados y registrados:

Paso I: Movimientos repetitivos.

Paso II: Postura/movimientos

Paso III: Fuerza

Paso IV: Tiempos de recuperación o descanso.

Los niveles de riesgo son categorizados con los siguientes colores:

- Verde: Significa que la condición observada no señala riesgo por lo cual se mantendrán las condiciones.

- Amarillo: Señala que existe el factor de riesgo en una criticidad media y debe ser corregido.

- Rojo: Señala que el factor de riesgo y la condición de exposición en el tiempo de evaluación son inaceptables o críticas y deberán ser corregidas.

\section{Análisis e interpretación de resulltadlos}

Tabla 1.-

Resultados de los factores de riesgo encontrados en la empresa Maxi-Distribuciones

\begin{tabular}{|l|c|c|c|c|}
\hline Zona & $\begin{array}{c}\text { Movimientos } \\
\text { repetitivos }\end{array}$ & $\begin{array}{c}\text { Postura/ } \\
\text { movimiento }\end{array}$ & Fuerza & $\begin{array}{c}\text { Tiempo de recuperación } \\
\text { o descanso }\end{array}$ \\
\hline Verde & 49 & 59 & 25 & 33 \\
\hline Amarillo & 66 & 72 & 28 & 45 \\
\hline Rojo & 10 & 14 & 3 & 9 \\
\hline TOTAL & 125 & 145 & 56 & 87 \\
\hline
\end{tabular}

Fuente: Elaboración propia 
Como resultado de la identificación de factores de riesgo relacionados con movimientos repetitivos, postura, fuerza y tiempos de recuperación que pueden tener los trabajadores de Maxi-Distribuciones durante la jornada laboral, se evidenció que: el nivel de riesgo de las condiciones observadas en el Paso I Movimientos repetitivos, en zona verde el 39\% del total del personal realiza movimientos repetitivos combinados en un periodo de cuatro horas de la jornada laboral con pausas de recuperación o descanso por cada hora de trabajo. Esta es una cifra importante porque estos trabajadores durante sus horas laborales realizan alternancia de movimientos rompiendo la continuidad y repeticiones por algunos instantes, estimulando y distiende las articulaciones móviles como: codo, rodilla, cuello, dedos, manos muñecas; usadas en movimientos idénticos o casi idénticos. Este grupo de trabajadores, empíricamente están utilizando algunos movimientos que forman parte de la técnica de las pausas activas.

De otra parte, un $52,8 \%$ de la población trabajadora se encuentra en un nivel de riesgo amarillo, es decir, que durante las cuatro horas consecutivas de trabajo mientras desarrollan sus tareas. Los movimientos realizados son repetitivos y parecidos, acompañados por esfuerzos estáticos, poco combinados con pausas de descanso los que van de hora y media ardos horas, ocasionando en el organismo del trabajador dolencias y alteraciones musculares, de tendones y ligamentos moderados que son perjudiciales para la salud.

En zona roja con un porcentaje del $8,2 \%$, sin embargo de ser menor a los dos anteriores, no deja de ser alarmante para este análisis porque si se mantiene esta condición la consecuencia a largo plazo es la aparición de lesiones permanentes y de cansancio prematuro.

Para el Paso II de Posturas / Movimientos se observa que en zona verde el $40,7 \%$ realizan pequeñas desviaciones de la posición normal de: dedos muñeca, codo, hombro, pierna, rodilla, pie y tronco. Las tareas se desarrollan en menos de cuatro horas, dándose leves descansos para recuperarse cada hora. Estos trabajadores tratan de compensar aquellas posturas fijas y de inactividad parándose, estirándose, realizándolo por inercia, cansancio o dolor leve.

En zona amarilla se registra un riesgo moderado del 49,65\%. Los trabajadores realizan escasas desviaciones o movimientos que alivien y balanceen las posturas repetidas durante las cuatro horas laborales evaluadas, lo cual afecta al aparato locomotor, forzando la estructura muscular de la espalda, antebrazo, mano, cuello y demás extremidades que se comprometan en la ejecución de la tareas cotidianas e imprevistas como acarreo y agarre abrupto de objetos, sin la adecuada flexión o extensión de las partes del cuerpo humano lo que puede ocasionar fatiga muscular, dolores, inflamaciones por estos movimientos anómalos y descoordinados. Para la zona roja estos trastornos pueden llegar a ser irreversibles, si no se toma las medidas correctivas y preventivas necesarias, esperando que no se supere la condición de riesgo irreversible del 9,6\% en esta zona.

El paso III se lo realizó a trabajadores que utilizan la fuerza física para la ejecución de la mayoría de sus tareas, mostrándose así que un 44,6\% empuja, arrastra o sostiene un objeto realizando una alternancia equilibrada de las articulaciones que se contraen y se extienden; es decir, la exigencia física está dentro de lo normal, pues la frecuencia del mismo nivel de fuerza es combinada con factores posturales y con tiempos de recuperación. Por cada dos horas de trabajo existe modificación de la postura corporal y de la intensificación de la fuerza.

En zona amarilla, el 50\% de los trabajadores ejercen intensas fuerzas al levantar o manipular objetos pesados sin variación correcta de la postura, lo que 
intensifica la fuerza y por ende el esfuerzo, trayendo consigo fallos en la estructura muscular. Estos esfuerzos prolongados pueden tornarse de leves a moderados o de moderados a severos, causando enfermedades degenerativas por estas dosis acumuladas de mucha tensión y poca relajación, que va de meses a años, esta enfermedad degenerativa, en el desarrollo de este tipo de actividades que requieren algún tipo fuerza, que afectará la región lumbar y extremidades superiores, deteriorando la funcionalidad. En esta misma condición observada no es muy alto el porcentaje de la zona roja, pero no deja de ser menos importante, el tomar medidas que corrijan y mejoren este factor que es de un 5,4\%.

Para finalizar, el análisis en el paso IV correspondiente a tiempos de recuperación o descanso, el 38\% de los trabajadores durante la jornada laboral mantienen un horario fijo para almorzar de mínimo treinta minutos, y un tiempo de recuperación de hasta quince minutos distribuidos entre la mañana y la tarde; en zona amarilla, el 52\% de los trabajadores tiene el mismo tiempo para almorzar pero lastimosamente por el exceso de trabajo, visitas a clientes, arreglos y mantenimientos del sistema troncalizado que brinda el servicio de radiocomunicación, estos trabajadores pasan por alto el tiempo del almuerzo, debido a la variación de horario, comen a deshoras, lo que es peligroso porque se puede producir un desbalance alimenticio, una deficiente energía corporal, dolores de cabeza que no permita desarrollar el trabajo adecuadamente.

Adicionalmente, el tiempo de recuperación de las actividades laborales es mal utilizado, pues realizan actividades como fumar, conversar, utilizar celulares y tablets que no ayudan a activar la circulación sanguínea, potenciar el funcionamiento cerebral y ejercitar músculos y tendones que sufren mayor impacto en la jornada laboral; sino a aumentar dolores e inflamaciones en dedos, manos, muñecas y brazos.
En zona roja un $10 \%$ de los trabajadores no dimensiona las posibles afectaciones que puede causar el no comer o engañar al estómago con alimentos poco nutritivos, además de no pausar por algunos minutos el trabajo para que el cuerpo se estire, mueva, relaje y se distraiga la mente.

Los resultados obtenidos en esta lista de chequeo muestran la existencia de condiciones de riesgo en la salud del trabajador; según la metodología propuesta por (Cerda Díaz, 2012) se evidencia que el más alto porcentaje se encuentra en zona amarilla, es decir, un riesgo moderado lo cual requiere una pronta acción para transformar esta condición a zona verde.

En esta zona verde el riesgo es leve o inexistente, pero no así menos importante, en este caso el porcentaje que se obtuvo se deberá fortalecer, a fin de mantenerlo o incrementarlo y no pase a zona de riesgo amarilla o roja.

Deberá darse atención inmediata a la zona roja que no presenta porcentaje alto, pero si preocupante. Esta es una condición de riesgo severa, de no intervenir podría trastornarse en perjudicial para la salud del trabajador y para la organización, afectando al rendimiento del trabajador y por ende a la producción de la organización para estos grupos vulnerables de trabajadores.

Las medidas a tomarse por parte de la gerencia y la unidad de talento humano tienden a desarrollar actitudes personales y corporativas saludables, que prevengan y promocionen hábitos saludables durante la jornada laboral, utilizando la técnica de las pausas activas a fin de redimir, mejorar y prevenir desordenes en el aparato locomotor y lo más importante como es el aportar al bienestar general y salud de los trabajadores en el desempeño de sus tareas que según (Davis, 1966) citado por (Chiavetano, 2009) se lo denomina calidad de vida laboral. 


\section{Aplicación dell programa dle pausas activas en Maxi-Distribuciones}

Esta técnica activa, alivia y compensa grupos musculares, movilidad articular y molestias osteo musculares a través de una rutina de ejercicios en el puesto de trabajo, por un espacio de 10 a 15 minutos durante la jornada laboral.

La práctica de este programa se lo realizó por cinco semanas consecutivas efectuándose tres sesiones de cinco minutos en la jornada laboral repartida al inicio, durante y al final de la jornada a la mayoría de los trabajadores; mientras que en el caso de los técnicos de montaje (Torristas) se realizó al inicio y al final de la jornada, para los trabajadores que desarrollan su trabajo en su mayoría fuera de las instalaciones de la empresa, dentro del programa se ejecutó esta rutina en los tres momentos de la jornada laboral.
La rutina para los trabajadores administrativos fue: cuello, hombros, dedos, manos, codos, ojos, espalda, abdomen, cadera y miembros inferiores.

Para los trabajadores del área técnica, la rutina que se aplicó fue: cuello, hombros, brazos, codos, espalda, abdomen, cadera, piernas y pie.

\section{Evaluación de los resultados}

La rutina de ejercicios propuestos como parte del programa de pausas activas ha contribuido a cada uno de los factores evaluados y presentados en el diagnóstico de la situación actual de la organización de la tabla $\mathrm{N}^{\mathrm{o}} 2$.

Para la evaluación de resultados del programa de pausas activas, se utilizó la misma metodología trabajada en el diagnóstico con que se determinó la situación inicial de los factores de riesgo.

Tabla 2.-

Contribución de la rutina de ejercicios en los factores de riesgo

\begin{tabular}{|l|c|c|c|}
\hline & $\begin{array}{c}\text { Movimientos } \\
\text { repetitivos }\end{array}$ & $\begin{array}{c}\text { Postura/ } \\
\text { movimiento }\end{array}$ & Fuerza \\
\hline Administrativos & & $\mathrm{X}$ & \\
\hline Cuello & $\mathrm{X}$ & $\mathrm{X}$ & \\
\hline Hombros & $\mathrm{X}$ & $\mathrm{X}$ & \\
\hline Dedos, manos y codos & & $\mathrm{X}$ & \\
\hline Ojos & $\mathrm{X}$ & $\mathrm{X}$ & \\
\hline Espalda y abdomen & & & $\mathrm{X}$ \\
\hline Cadera y miembros inferiores & & $\mathrm{X}$ & \\
\hline Técnicos & & $\mathrm{X}$ & \\
\hline Cuello & $\mathrm{X}$ & $\mathrm{X}$ & \\
\hline Hombros & $\mathrm{X}$ & $\mathrm{X}$ & $\mathrm{X}$ \\
\hline Brazos y codos & & $\mathrm{X}$ & $\mathrm{X}$ \\
\hline Espalda y abdomen & & & \\
\hline Caderas, piernas y pies & & & \\
\hline
\end{tabular}

Fuente: Elaboración propia 
La evaluación de la lista de chequeo, permitió registrar (observación directa) redimensionar y reubicar el número de movimientos repetitivos, postura/movimiento, fuerza, tiempo de recuperación o descanso, luego de haber ejecutado el programa de pausas activas. Se realizó un comparativo entre la situación inicial o diagnóstico y la posterior a la utilización del programa. Los resultados se presentan en el gráfico 1.

\section{Gráfico 1.}

Cuadro comparativo con el programa de pausas activas y sin pausas.

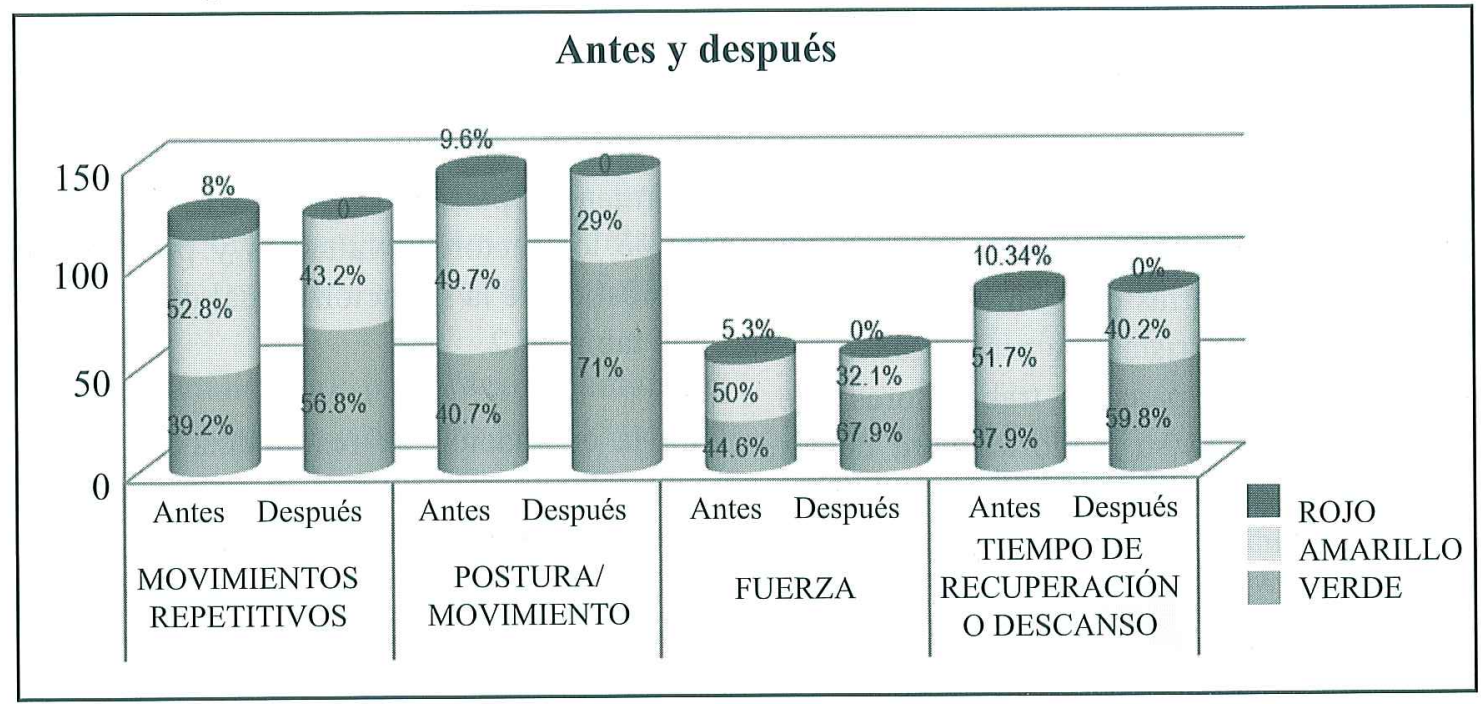

Fuente: Elaboración propia

En la evaluación de los resultados, en forma general, se aprecia cambios significativos en beneficio de la salud laboral de los trabajadores de MaxiDistribuciones, lo que permite aportar a la mejora de la calidad de vida laboral de los mismos. Situación que se evidencia en la eliminación de los riesgos considerados como severos, que son los factores de riesgo representados en el gráfico, con color rojo; que, comparados entre la condición observada en el diagnóstico y la condición actual, han mostrado una disminución importante.

\section{Conclusiomes}

La solución al problema planteado se ha reflejado significativamente en la concienciación adquirida por los trabajadores y la gerencia de MaxiDistribuciones. La práctica de las pausas activas, ha contribuido en la salud laboral y en la calidad de vida laboral.

En el marco teórico se presentó la importancia de la práctica de programas de prevención y promoción de la salud laboral. Se documentó la utilidad de las pausas activas como instrumento para reducir afecciones laborales causadas por el uso constante de tecnología, lo cual acarrea problemas de salud, como: lumbalgias, síndromes de trauma acumulativo, túnel carpiano y fatiga visual.

La metodología aplicada, permitió identificar los factores de riesgo relacionados a posturas, 
movimientos repetitivos, fuerza y descansos pasivos; también, el nivel de riesgo observado en el puesto de trabajo. El diagnóstico presentó las condiciones habituales en el trabajo que generan deterioro potencial en la salud de los trabajadores de Maxi-Distribuciones, los que son causantes de altos porcentajes de riesgo por posturas estáticas e inadecuadas, poca variación de actividades, descansos mal utilizados y fuerzas que no tienen la compensación muscular necesaria, afectando al bienestar laboral.

A partir de la utilización del programa de pausas activas en la empresa Maxi-Distribuciones, el impacto fue favorable en la salud y rendimiento de los trabajadores. Se dio un crecimiento porcentual de la zona verde, en donde, los niveles de riesgo pasan de leves a inexistentes, evolucionando esta zona en 17.6, 30.3, 23.3 y $21.6 \%$ en movimientos repetitivos, postura/movimiento, fuerza y tiempo de recuperación o descanso respectivamente. Esto significa que en estos porcentajes se redujeron el riesgo en zona amarilla y la eliminación total del riesgo en la zona roja.

La implementación de las rutinas de ejercicios consideradas en el programa de pausas activas tuvo una acogida inmediata por ser sencillas y útiles para la relajación, calentamiento y estiramiento de articulaciones móviles; músculos y extremidades que más se esfuerzan durante la jornada laboral. Se generó en los trabajadores estilos saludables y de auto cuidado, sintiéndose más motivados y satisfechos por el grado de interés y preocupación, demostrado por el empleador en promover y promocionar programas que prevengan y mejoren la salud.

Se puede detallar los logros alcanzados con la utilización del programa de pausas activas: elevó el rendimiento laboral de los trabajadores; elevó la actitud y disminuyó la apatía e indiferencia del trabajador en el desempeño de sus labores; redujo los costos del personal; disminución notable del ausentismo temporal por causas relativas a enfermedades no trasmisibles, trayendo como efecto, la disminución de la rotación del personal en áreas técnicas. Este conjunto de logros ha contribuido finalmente a la salud y calidad de vida laboral de los trabajadores de Maxi-Distribuciones.

La receptividad dada a la propuesta del programa de pausas activas, por parte de los cargos directivos, demostró el interés de combatir riesgos laborales que pueden afectar negativamente a la calidad de vida laboral, percibida por el trabajador en la organización. 


\section{Referencias biblliográficas}

- Castro E, M. J. (2011). Efectos de un programa de pausas activas sobre la persepción de desórdenes músculoesqueléticos en trabajadores de la universidad de Antoquia. Recuperado el 28 de junio de 2014, de http:// aprendeenlinea.udea.edu.co/revistas/index.php/educacionfisicaydeporte/article.

- Cerda D, L. (2012). Norma Técnica de Identificación y Evaluación de Factores de Riesgo Musculoesqueléticos. Obtenido de http://web.minsal.cl/portal/url/item/cbb583883dbcle79e040010165014f3c.pdf

- Cerda Díaz, L. (2012). Norma Técnica de Identificación y Evaluación de Factores de Riesgo Musculoesqueléticos. Obtenido de http://web.minsal.cl/portal/url/item/cbb583883dbc1e79e040010165014f 3c.pdf

- Chiavetano, I. (2009). Gestión del talento humano. Mexico: Mc Graw Hill.

- Davis, L. E. (1966). The Desing of Jobs. Industrial Relations.

- Delgado J, U. D. (01 de noviembre de 2014). Gestión del talento humano como herramienta de competitividad en las empresas. Obtenido de http://webquery.ujmd.edu.sv/siab/bvirtual/Fulltext/ADAG0000538/ Capitulo\%205.pdf

- El Comercio. (07 de junio de 2014). Sección Tendencias. Cinco enfermedades más comunes en el trabajo.

- Guerrero J, A. M. (2004). Salud ocupacional: nociones útiles para los profesionales de la información. Recuperado el 01 de 12 de 2014, de http://bvs.sld.cu/revistas/aci/vol12_5_04/aci05504.htm

- Logroño L, D. L. (2011). Salud ocupacional prevención de riesgos laborales. seguridad y salud en el trabajo. Edición 1, 55.

- Mazzafero E, V. (1999). Medicina y salud pública. Buenos Aires: Eudeba.

- Ministerio de Salud, P. (2013). Somossalud. Recuperado el 12 de agosto de 2014, de http://somossalud.msp. gob.ec/index.php/agita-tu-mundo/

- Ministerio del Deporte, E. (2012). Ministerio del Deporte realiza pausa activa laboral en instituciones públicas de Esmeraldas. Recuperado el 12 de agosto de 2014, de http://www.deporte.gob.ec/ministerio-deldeporte-realiza-pausa-activa-laboral-en-instituciones-publicas-de-esmeraldas/

- Mossink J, J. C. (2004). Prevención de transtornos musculoesqueléticos en el lugar del trabajo. En $\mathrm{N}^{\circ} 5$ Serie de protección salud de los trabajadores (pág. 8). Francia: Instituto Federal de Salud Ocupacional.

- OIT. (2010). Lista de Enfermedades Profesionales. Ginebra: Serie de Seguridad y Trabajo N74.

- Oliveira, J. (2006). A Prática da Ginástica Laboral $3^{\mathrm{a}}$ ed. Rio de Janeiro: Sprint.

- OMS. (2014). Obtenido de Organizacion Mundial de la Salud: http://www.who.int/countries/ecu/es/

- Parra M, M. (2003). Conceptos básicos en salud laboral. Santiago de Chile: Publicaciones Oficina Internacional de Trabajo.

- Salud, A. E. (02 de 12 de 2013). Desayunos de salud. Obtenido de Promoción de la salud laboral: menos absentismo y más competitividad: http://www.efesalud.com/noticias/promocion-de-la-salud-laboral-menosabsentismo-y-mas-competitividad/

- Salud, O. M. (1995). Estrategia Mundial de la Salud Ocupacional Para Todos. Obtenido de apps.who.int/ iris/bitstream/10665/42109/1/951802071X_spa.pdf

- Salud, O. M. (2003). Salud Ocupacional - Promocion de la salud. Boletin de la Gohnet No6 The Global Occupational Health Network. 